\title{
Transmission properties of double- gap asymmetric split ring resonators in terahertz region
}

\section{$\operatorname{AUTHOR}(\mathrm{S}):$}

Miyamaru, F.; Kubota, S.; Nakanishi, T.; Kawashima, S.; Sato, N.; Kitano, M.; Takeda, M. W.

\section{CITATION:}

Miyamaru, F. ... [et al]. Transmission properties of double-gap asymmetric split ring resonators in terahertz region. Applied Physics Letters 2012, 101(5): 051112.

\section{ISSUE DATE:}

2012-08-02

URL:

http://hdl.handle.net/2433/187969

\section{RIGHT:}

(c) 2012 American Institute of Physics. This article may be downloaded for personal use only. Any other use requires prior permission of the author and the American Institute of Physics. 


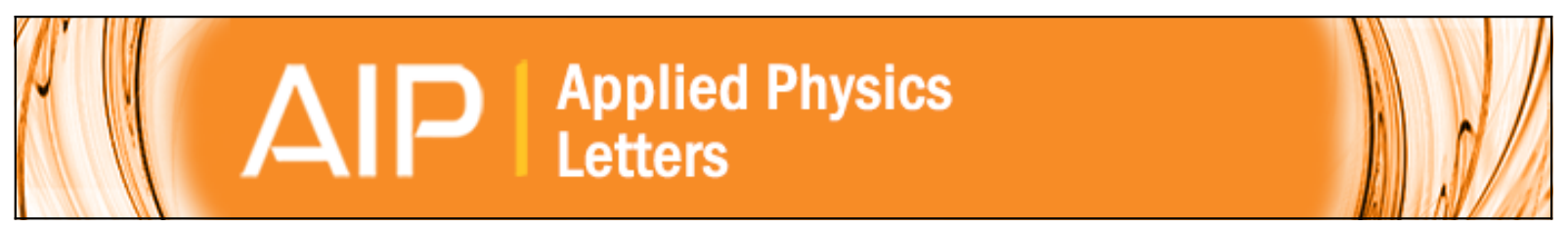

\section{Transmission properties of double-gap asymmetric split ring resonators in terahertz region}

F. Miyamaru, S. Kubota, T. Nakanishi, S. Kawashima, N. Sato, M. Kitano, and M. W. Takeda

Citation: Applied Physics Letters 101, 051112 (2012); doi: 10.1063/1.4739945

View online: http://dx.doi.org/10.1063/1.4739945

View Table of Contents: http://scitation.aip.org/content/aip/journal/apl/101/5?ver=pdfcov

Published by the AIP Publishing

\section{Articles you may be interested in}

Dual-band terahertz metamaterials based on nested split ring resonators

Appl. Phys. Lett. 101, 091103 (2012); 10.1063/1.4748163

Excitation of a high-Q subradiant resonance mode in mirrored single-gap asymmetric split ring resonator terahertz metamaterials

Appl. Phys. Lett. 101, 071108 (2012); 10.1063/1.4745790

Broadband polarization transformation via enhanced asymmetric transmission through arrays of twisted complementary split-ring resonators

Appl. Phys. Lett. 99, 221907 (2011); 10.1063/1.3664774

Highly selective left-handed transmission line loaded with split ring resonators and wires

Appl. Phys. Lett. 94, 143503 (2009); 10.1063/1.3116647

Properties of a metamaterial element: Analytical solutions and numerical simulations for a singly split double ring J. Appl. Phys. 95, 3778 (2004); 10.1063/1.1652251

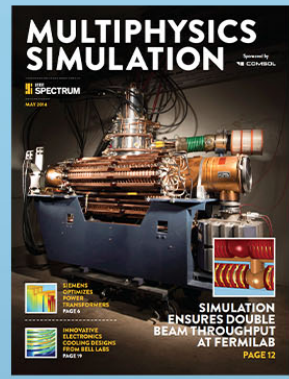

Free online magazine

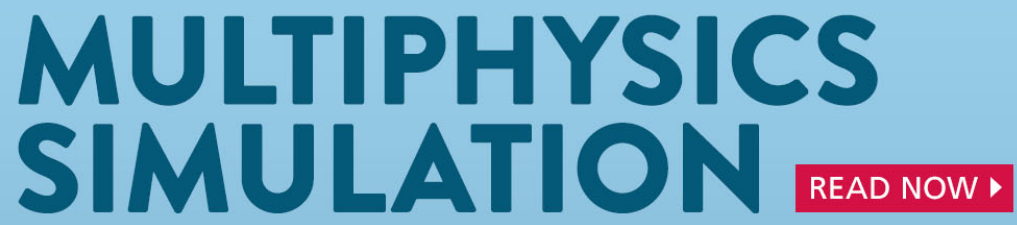




\title{
Transmission properties of double-gap asymmetric split ring resonators in terahertz region
}

\author{
F. Miyamaru, ${ }^{1}$ S. Kubota, ${ }^{1}$ T. Nakanishi, ${ }^{2}$ S. Kawashima, ${ }^{1}$ N. Sato, ${ }^{1}$ M. Kitano, ${ }^{2}$ \\ and M. W. Takeda ${ }^{1}$ \\ ${ }^{1}$ Department of Physics, Faculty of Science, Shinshu University, 3-1-1 Asahi, Matsumoto, Nagano, Japan \\ ${ }^{2}$ Department of Electronic Science and Engineering, Kyoto University, Kyoto 615-8510, Japan
}

(Received 13 January 2012; accepted 16 July 2012; published online 2 August 2012)

\begin{abstract}
We investigated the electromagnetic properties of the metamaterials that consist of double-gap split ring resonators (SRRs) in the terahertz region. We found that varying the position of one gap with respect to the other causes the resonant frequency of the SRRs to shift over a broad range. This frequency shift is attributed to the change in the combined capacitance that consists of two capacitances of gaps connected in series and an additional capacitance connected in parallel to the others. Our findings are also verified by obtaining good agreement between experiments and simulations. (C) 2012 American Institute of Physics. [http://dx.doi.org/10.1063/1.4739945]
\end{abstract}

Manipulating and controlling light with metamaterials, ${ }^{1}$ which consist of subwavelength artificial structures, are very attractive for the purpose of fabricating optical devices. Attempts to produce extraordinary optical phenomena by using metamaterials have been demonstrated, including negative refractive index materials ${ }^{2}$ and transformation optics. ${ }^{3}$ Many studies about metamaterials have used resonant elements as unit cells to induce electric and magnetic resonance. A split ring resonator (SRR) is a promising structure that can be used to obtain magnetic resonance.

The reflection and transmission characteristics of SRRs are determined by their sizes and shapes. Until now, many studies about tuning or switching the electromagnetic properties of SRRs have been reported. ${ }^{4-11}$ For example, Singh et al. investigated the terahertz $(\mathrm{THz})$ transmission characteristics of asymmetric SRRs by varying the position of the capacitive gap and found that the frequency of the resonant dip can be modulated. ${ }^{10}$ Such a modulation of electromagnetic properties is very useful not only for passive THz metamaterials but also for $\mathrm{THz}$ active photoconductive antennas loaded with SRRs. ${ }^{12}$ Recently, Takano et al. reported on resonant $\mathrm{THz}$ emissions from SRR-loaded photoconductive antennas and demonstrated control of the emissions' properties by pumping the capacitive gap of the SRR with femtosecond optical pulses.

In this paper, we investigate the transmission characteristics of SRRs with double capacitive gaps. By varying the position of one of the capacitive gaps with respect to the other, we observe a broad range of frequency shifts of the transmission dip. We simulate the current distributions for our SRRs and attribute the mechanism of this frequency shift to the change of the combined capacitance of the SRRs. This characteristic of the double-gap SRRs makes possible the fabrication of switchable $\mathrm{THz}$ photoconductive antennas with broad range resonant frequency tuning.

Figure 1 shows diagrams of SRRs with the double capacitive gaps used in our study. We set the length of one side to $a=100 \mu \mathrm{m}$ and the gap width to $b=20 \mu \mathrm{m}$ for all SRRs. The position of one of the gaps is varied with respect to the other with displacement parameters $\delta_{y}$ and $\delta_{x}$ that are measured from the top side for SRR1 $\left(\delta_{y}=14 \mu \mathrm{m}\right)$, SRR2 $\left(\delta_{y}=50 \mu \mathrm{m}\right)$, and SRR3 $\left(\delta_{y}=86 \mu \mathrm{m}\right)$; and the left side for SRR4 $\left(\delta_{x}=14 \mu \mathrm{m}\right), \quad$ SRR5 $\left(\delta_{x}=50 \mu \mathrm{m}\right), \quad$ and $\quad$ SRR6 $\left(\delta_{x}=86 \mu \mathrm{m}\right)$, respectively. Both the line width and line thickness are $2 \mu \mathrm{m}$. We simulate the transmission spectra of the SRRs by using the finite difference time domain (FDTD) method in three-dimensional space. SRRs are arranged with square lattice structure (lattice constant $p=150 \mu \mathrm{m}$ ) in two dimensions of the $y-z$ or $x-z$ plane depending on the propagation direction $\boldsymbol{k}$ of the incident wave. When the incident wave propagates along $x(y)$ axis, SRRs are arranged periodically in $y-z(x-z)$ plane. The incident magnetic field $\boldsymbol{B}$ is directed perpendicular to the SRR plane in order to induce a circular current and hence the magnetic resonance, while the

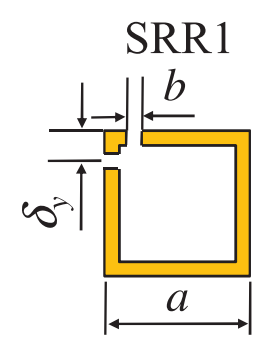

\section{SRR4}
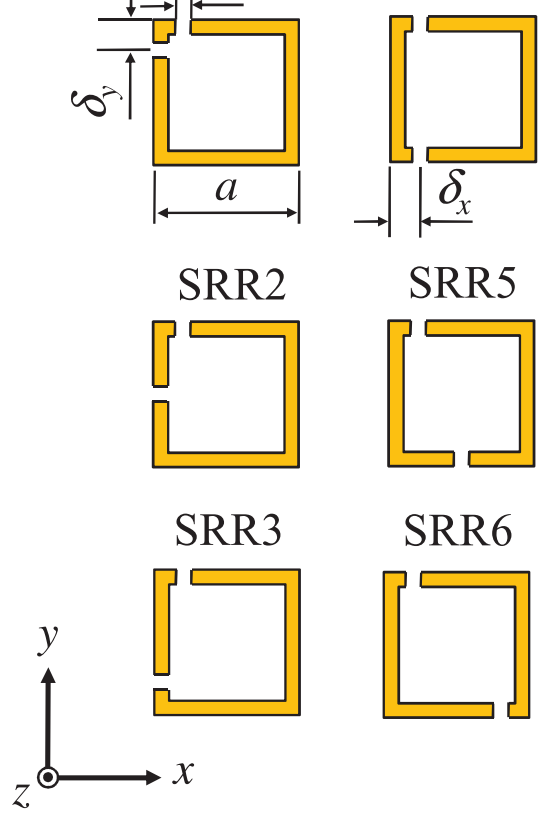

FIG. 1. Diagrams of double-gap SRRs used in our study. The geometrical parameters are $a=100 \mu \mathrm{m}$ and $b=20 \mu \mathrm{m}$ for all SRRs. 
(a)

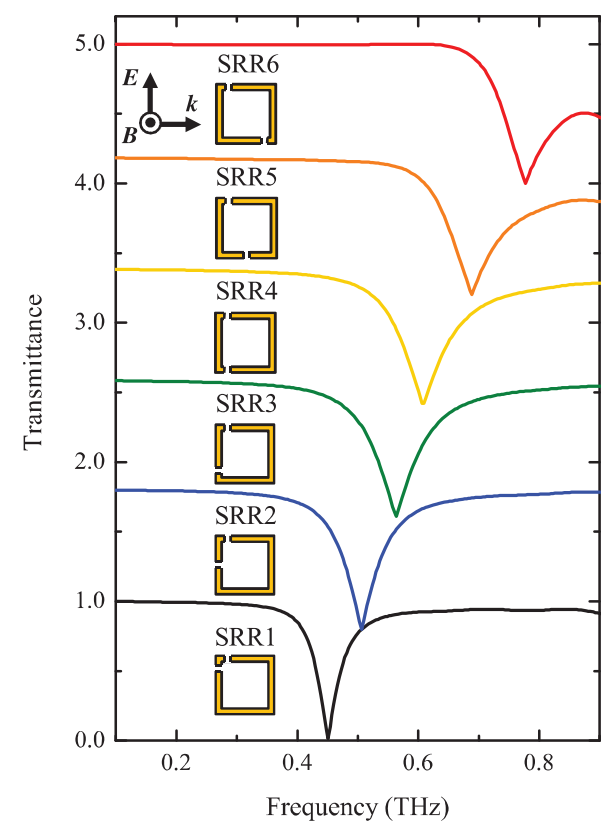

(b)

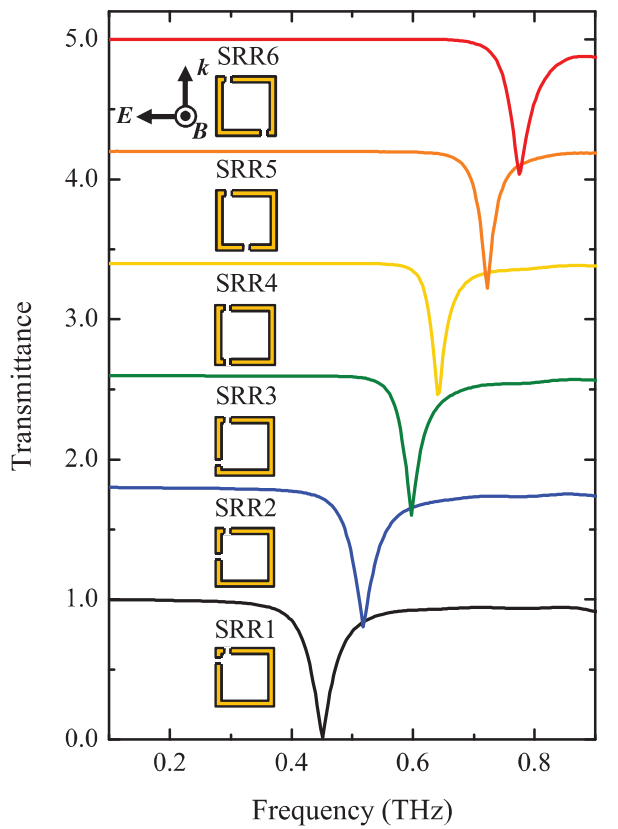

FIG. 2. Simulated transmission spectra for SRR1-SRR6 shown in Fig. 1. Incident electromagnetic wave is illuminated from (a) left and (b) bottom of SRRs as shown in inset. Directions of incident electric and magnetic field are also shown in inset. Each spectrum is shifted in the vertical direction for clarity. direction of the incident electric field $\boldsymbol{E}$ depends on the propagation direction of the incident wave. In this simulation, we assumed a perfect conductor for the material of the SRRs.

Figures 2(a) and 2(b) show the simulated transmission spectra of the double-gap SRRs for different propagation directions of the incident wave as shown in inset of each figure. Each spectrum is shifted in the vertical direction for clarity. For SRR1 in Fig. 2(a), the transmission dip is observed at $0.45 \mathrm{THz}$, which is attributed to the LC resonance of the SRR. ${ }^{11}$ The transmission dip shows a blue shift as one of the capacitive gaps moves away from the other fixed gap (from SRR1 to SRR6), and reaches approximately $0.75 \mathrm{THz}$ for SRR6. The amount of this resonant frequency shift is much larger than that observed in the case of the single gap displacement. ${ }^{10}$ This characteristic could be very useful for tuning the resonant frequency of $\mathrm{THz}$ emissions in applications using double-gap SRRs as loaded elements for a photoconductive THz antenna. ${ }^{12}$ The shape of the transmission dips looks asymmetric for SRR5 and SRR6. This is because another resonant dip, which is attributed to the electric resonance, is near the LC resonant frequency.

In Fig. 2(b), the resonant frequency shows the similar blue-shift to that observed in Fig. 2(a). However, some discrepancies of the bandwidth and resonant frequency are observed between Figs. 2(a) and 2(b). These discrepancies might be attributed to the difference of the incident electric field polarization. In an asymmetric optical configuration of SRRs, the incident electric field induces the circular current through the bianisotropic effect. ${ }^{11}$ In our SRRs, the strength of this bianisotropic effect depends on the shape of the SRR and the electric polarization. Additionally, the electrically induced circular current interacts with the other circular current that is induced by the incident magnetic field normal to the SRR plane. As the result, the transmission dip shows different frequency and spectral shape depending on the incident electric field polarization.
Next, we investigate the mechanism of the frequency shift of the SRRs in detail. In Fig. 3, we show the calculated current (arrows) and electric charge (color image) distributions for SRR1-SRR6, respectively, at each resonant frequency. For this calculation, we used the HFSS frequency domain solver. All figures show the distributions at same phase where both the current and electric charge distributions are clearly observed. Phase variations of the current and electric charge distributions for each SRR are also given as multimedia movie files. In SRR1, circular current flow can be observed along the longer metallic segment. This type of current distribution is also observed in SRR2 and SRR3. Only very weak current is observed along the shorter metallic segment in SRR3. With increasing $\delta_{x}$ for SRR4SRR6, and correspondingly as the lengths of the two metallic segments become more similar, the magnitude of the current flow along the shorter metallic segment increases gradually. For SRR6, the lengths of the two metallic segments are the same, and the current distribution becomes symmetric.

From the simulated results of the current distributions, the mechanism of the frequency shift observed in Fig. 2 is considered as follows. Figure 4(a) shows a diagram of the equivalent capacitance of SRR3. In addition to $\mathrm{C}_{1}$ and $\mathrm{C}_{2}$, which are connected in series, the additional capacitances $C_{3}$ and $\mathrm{C}_{4}$ that are connected in parallel with respect to the other capacitances should be considered. The current distributions can be roughly classified into two cases, depending on $\mathrm{C}_{3}$ and the combined capacitance of $\mathrm{C}_{1}$ and $\mathrm{C}_{2}$, i.e., $\mathrm{C}_{12}=\mathrm{C}_{1}$ $\mathrm{C}_{2} /\left(\mathrm{C}_{1}+\mathrm{C}_{2}\right)$. For SRR1, SRR2, and SRR3, $\mathrm{C}_{3}$ is much larger than the combined capacitance $C_{12}$, and therefore, the loop current is formed by the electric current along the longer metallic segment and displacement current through $\mathrm{C}_{3}$, as observed in Fig. 3. As the distance between the gaps increases from SRR3 to SRR6, the value of $\mathrm{C}_{3}$ decreases, and the current in the short metallic segment increases owing to the displacement current through $\mathrm{C}_{12}$. Although all the circuit parameters including $\mathrm{C}_{4}$ or the inductances of the 
(a)

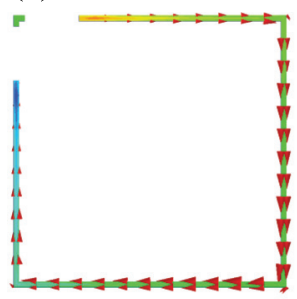

(b)

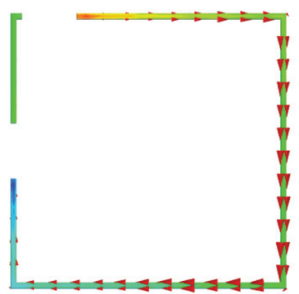

(c)

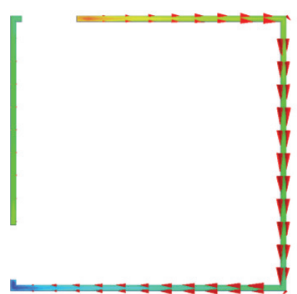

(d)

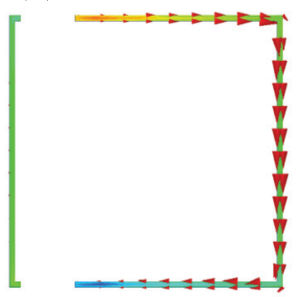

(e)

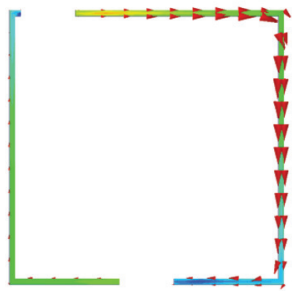

(f)

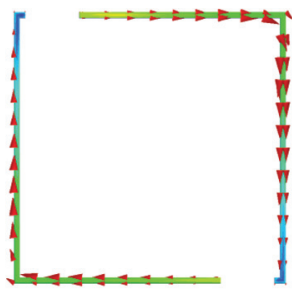

FIG. 3. Simulated distributions of surface current flow (arrow) and electric charge (color image) for SRR1-SRR6 at each resonant frequency. All figures are depicted at same phase for each resonant frequency (enhanced online) [URL: http://dx.doi.org/10.1063/1.4739945.1] [URL: http://dx.doi.org/ 10.1063/1.4739945.2] [URL: http://dx.doi.org/10.1063/1.4739945.3] [URL: http://dx.doi.org/10.1063/1.4739945.4] [URL: http://dx.doi.org/10.1063/ 1.4739945.5] [URL: http://dx.doi.org/10.1063/1.4739945.6].

metallic segment are required to obtain the absolute value of the resonant frequency, the blue shifts in the resonant frequencies can be qualitatively explained by the decreases of $\mathrm{C}_{3}$.

In order to verify the above discussion, we simulated the transmission spectra of SRR3 with three different values of $\mathrm{C}_{1}$ and $\mathrm{C}_{2}$, or $\mathrm{C}_{12}$. The calculated results are shown in Figs. 4(b) and 4(c) for each propagation direction of the incident wave as shown in insets. The red curve in Fig. 4(b) indicates the transmission spectrum of SRR3, which shows the transmission dip at $0.43 \mathrm{THz}$. Even if we remove the smaller segment from this SRR3 (black curve in Figs. 4(b) and 4(c)), the transmission spectrum is almost the same as the red curve. Only a very slight blue shift can be observed. This indicates that $\mathrm{C}_{3}$ is the dominant factor in the combined capacitance for SRR3. When $\mathrm{C}_{12}$ increases, on the other hand, the resonant frequency changes. The blue curves in Figs. 4(b) and 4(c) show the transmission spectrum of SRRs whose geometrical parameters are shown in the insets of each figure. The widths of both capacitive gaps are decreased to $4 \mu \mathrm{m}$ by increasing the length of the shorter metallic segment. This means that only $\mathrm{C}_{12}$ increases, and $\mathrm{C}_{3}$ is fixed. Owing to the increase of $\mathrm{C}_{12}$, and correspondingly the increase of the combined capacitance, we can observe that the resonant frequency shifts lower on the blue curve compared to the red curve. This result indicates that $\mathrm{C}_{12}$ gives relatively large contributions to the resonant characteristic. It is noted that

(a)

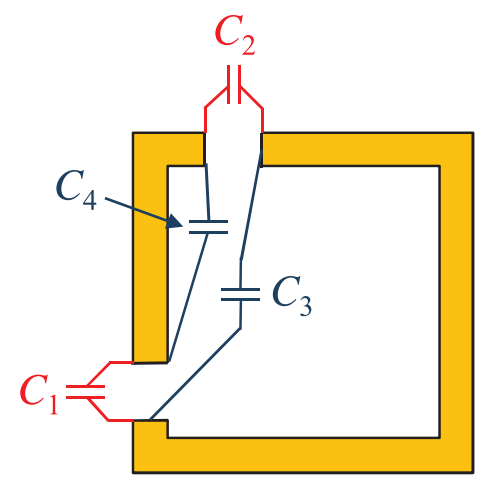

(b)

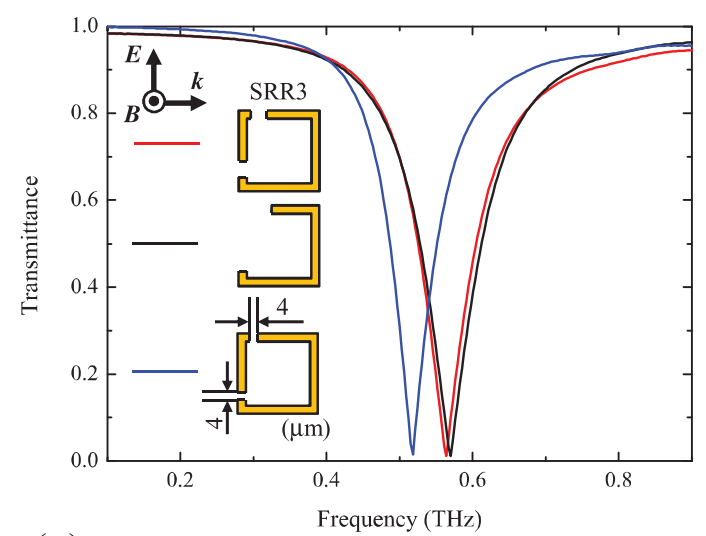

(c)

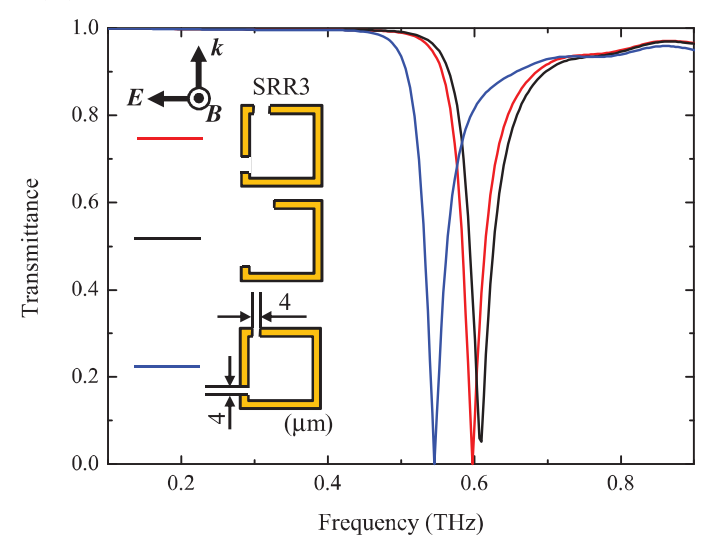

FIG. 4. (a) Diagram of double-gap SRR with equivalent capacitances. (b) and (c) Simulated transmission spectra for three types of SRRs. Inset of each figure shows the diagrams of SRRs used in this simulation and the configuration of the incident electromagnetic wave. For each figure, red line shows the transmission spectrum of SRR3. Black line is for the SRR that consists of only longer metallic segment of SRR3. Blue line is for the SRR that is same with SRR3 except the gap width of $4 \mu \mathrm{m}$.

we should also consider the near-field coupling between two metallic line segments as a contributing factor to the frequency shift. When the two resonators are placed enough closely with each other, the shift of each resonant frequency occurs as a result of the near-field coupling. ${ }^{13}$ This effect increases with decreasing the gap, and hence the distance between two metallic line segments becomes closer as observed in Figs. 4(b) and 4(c).

The contribution of $\mathrm{C}_{12}$ also becomes larger as the geometry of SRR changes from SRR1 to SRR6. Figure 5 shows the frequency shift of the resonant dip between our original 


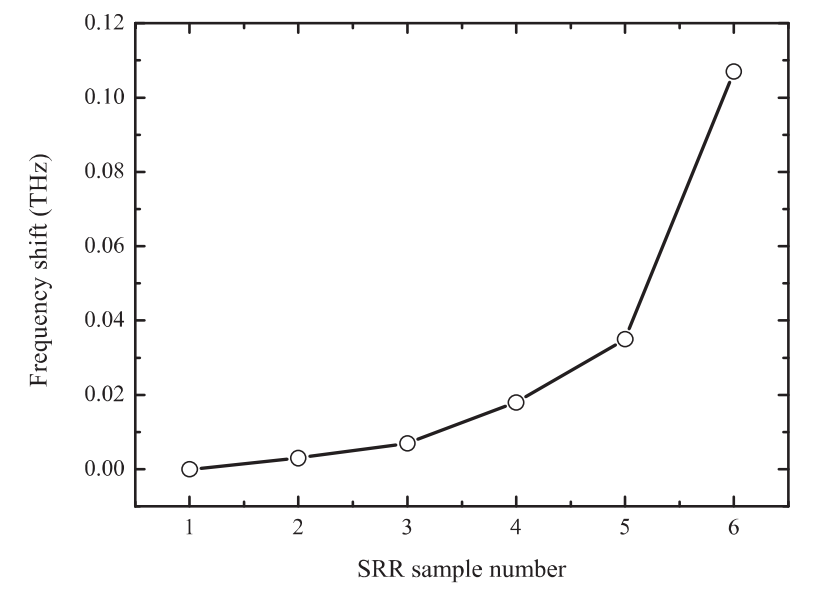

FIG. 5. Shift of resonant frequency between our original SRRs shown in Fig. 1 (SRR1-SRR6) and SRRs that consist of only longer metallic segment of each original SRR as a function of number of SRRs.

SRRs as shown in Fig. 1 (SRR1-SRR6) and SRRs that consist of only longer metallic segment of each original SRRs as a function of number of SRRs. For example, we plot the frequency shift $(0.007 \mathrm{THz})$ between dip frequencies of red $(0.563 \mathrm{THz})$ and black lines $(0.570 \mathrm{THz})$ in Fig. $4(\mathrm{c})$ as a value at SRR3. The propagation direction of the incident electromagnetic wave is same to that of Fig. 4(c). As the geometry of SRR changes from SRR1 to SRR6, the value of the frequency shift gradually increases, and reaches about $0.1 \mathrm{THz}$ for SRR6. This result indicates that the contributions of $\mathrm{C}_{12}$ gradually increases, and give very large contributions for SRR6.

Finally, we derive the experimental evidence for our finding in simulations. As shown in the inset of Fig. 6(a), we fabricated two metamaterials of double-gap SRRs with different gap positions on $100-\mu$ m-thick polyethylene terephthalate (PET) films. The geometrical parameters of these metamaterials are also shown in the figure. We used $\mathrm{THz}$ time-domain spectroscopy ${ }^{14}$ to measure the transmission spectrum with the incident $\mathrm{THz}$ wave propagating in the direction normal to the SRR plane. In our simulation, the incident magnetic field is directed normal to the SRR plane. However, since the measurement of the transmission spectrum is difficult in the optical configuration as performed in our simulation, we measure the transmission spectra at normal incidence in this experiment. In order to induce the LC resonance efficiently with the bianisotropic effect at normal incidence, the incident $\mathrm{THz}$ wave was polarized along the $y$ axis indicated in the inset of the Fig. 6(a). The black and red lines in Fig. 6(a) are transmission spectra for the metamaterials whose geometries are similar to those of SRR2 and SRR5 in Fig. 1, respectively. We can observe the apparent difference in the resonant dip frequency for the two samples. Figure 6(b) shows the simulated transmission spectra for two metamaterials with the same geometrical parameters. Good agreement between experiments and simulations is observed. These results give the experimental evidence that the resonant frequency of the double-gap SRR can be tuned by changing the gap positions. However, the observed dip frequencies of the LC resonance are lower than those observed in Figs. 2(a) and 2(b). These discrepancies are mainly due to
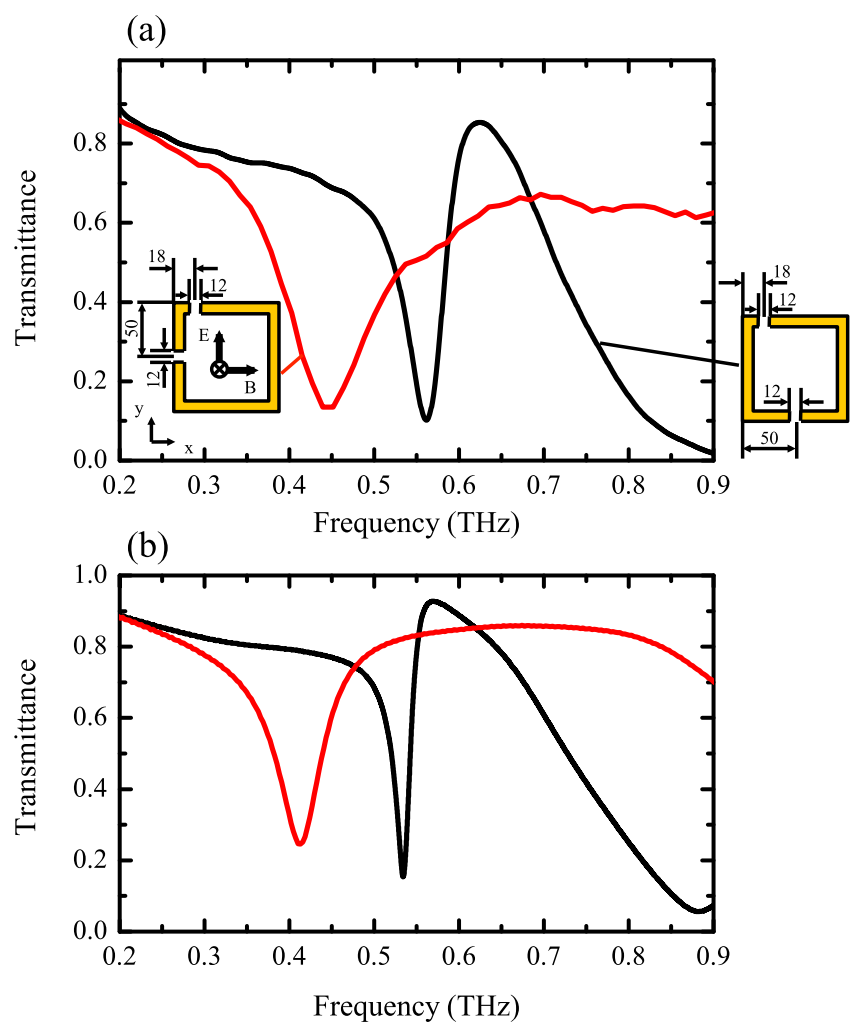

FIG. 6. (a) Measured and (b) simulated transmission spectra for double-gap SRRs of different geometrical parameters at normal incidence. Insets of (a) show the geometrical parameters of the SRRs.

the existence of PET film as substrate. They also are attributed to slight difference of the geometric parameters with respect to those of simulations owing to the limit of precision of our fabrication process.

In summary, we investigated the transmission characteristics of double-gap SRRs in the THz region both theoretically and experimentally. We found that the resonant frequency of the LC resonance can be shifted over a broad range by changing the position of one capacitive gap with respect to the other. From the results of the current distribution simulation, these results can be attributed to the variation of the combined capacitance that consists of two capacitances of gaps connected in series and an additional capacitance connected in parallel to the others. Such a broadband tuning of the LC resonant frequency is quite useful for applications involving resonant emissions of $\mathrm{THz}$ radiation from SRR-loaded photoconductive antenna devices.

This work was supported partially by the Ministry of Education, Science, Sports and Culture, Grant-in-Aid for Scientific Research (A) No. 20244047, Grant-in-Aid for Scientific Research on Innovative Areas No. 22109003, Grantin-Aid for Young Scientists (B) No. 21760036, the Izumi Science and Technology Foundation, and the Sumitomo Foundation.

\footnotetext{
${ }^{1}$ J. B. Pendry, A. J. Holden, D. J. Robbins, and W. J. Stewart, IEEE Trans. Microwave Theory Tech. 47, 2057 (1999).

${ }^{2}$ J. B. Pendry, Phys. Rev. Lett. 85, 3966 (2000).

${ }^{3}$ J. B. Pendry, D. Schurig, and D. R. Smith, Science 312, 1780 (2006).

${ }^{4}$ J.-M. Manceau, N.-H. Shen, M. Kafesaki, C. M. Soukoulis, and S. Tzortzakis, Appl. Phys. Lett. 96, 021111 (2010).
} 
${ }^{5}$ Z. C. Chen, M. H. Hong, C. S. Lim, N. R. Han, L. P. Shi, and T. C. Chong, Appl. Phys. Lett. 96, 181101 (2010).

${ }^{6}$ Y. Yang, R. Huang, L. Cong, Z. Zhu, J. Gu, Z. Tian, R. Singh, S. Zhang, J. Han, and W. Zhang, Appl. Phys. Lett. 98, 121114 (2011).

${ }^{7}$ R. Singh, E. Plum, W. Zhang, and N. I. Zheludev, Opt. Express 18, 13425 (2010).

${ }^{8}$ R. Singh, I. A. I. Al-Naib, M. Koch, and W. Zhang, Opt. Express 19, 6312 (2011).

${ }^{9}$ R. Singh, I. A. I. Al-Naib, Y. Yang, D. R. Chowdhury, W. Cao, C. Rockstuhl, T. Ozaki, R. Morandotti, and W. Zhang, Appl. Phys. Lett. 99, 201107 (2011).
${ }^{10}$ R. Singh, I. A. I. Al-Naib, M. Koch, and W. Zhang, Opt. Express 18, 13044 (2010).

${ }^{11}$ W. J. Padilla, A. J. Taylor, C. Highstrete, M. Lee, and R. D. Averitt, Phys. Rev. Lett. 96, 107401 (2006).

${ }^{12}$ K. Takano, Y. Chiyoda, T. Nishida, F. Miyamaru, T. Kawabata, H. Sasaki, M. W. Takeda, and M. Hangyo, Appl. Phys. Lett. 99, 161114 (2011).

${ }^{13}$ R. Singh, C. Rockstuhl, and W. Zhang, Appl. Phys. Lett. 99, 241108 (2010).

${ }^{14}$ M. Hangyo, T. Nagashima, and S. Nashima, Meas. Sci. Technol. 13, 1727 (2002). 